\title{
$\mathrm{AHP}$ 를 활용한 서비스 컴포지션 대상 선정에 대한 연구
}

\author{
김 지 혁 ${ }^{\dagger}$ 변 정 원 $^{++} \cdot$ 류 성 열 $^{+++}$
}

요 약

$\mathrm{SOA}$ 의 출현은 비즈니스 및 IT 환경에 많은 영향을 주었고 학계 및 산업에서 많은 연구가 진행 중이다. 향후 서비스는 폭발적으로 증가할 것이며, 서비스를 컴포지션하여 비즈니스 기회를 창출하고 IT는 이를 지원할 것이다. 그러나 컴포지션의 대상이 되는 서비스를 선정하는 연구 는 부족한 실정이다. 본 연구는 서비스 컴포지션 대상을 선정하는 프레임워크를 제안하는 것이며, 선정 방법으로는 Analytic Hierarchy Process 기법을 활용한다. 본 연구의 결과는 서비스 조합시 서비스의 기능/비기능 속성을 반영하고 다양한 이해당사자의 관점과 다수 선정 기준을 적용 하여 서비스 선정을 가능하게 하였다.

키워드 : 서비스, 컴포지션, Analytic Hierarchy Process

\section{A Study on Selection of a Service Composition Target using AHP}

\author{
Ji Hyeok Kim ${ }^{\dagger}$ J Jung Won Byun ${ }^{\dagger+} \cdot$ Sung Yul Rhew ${ }^{\dagger++}$
}

\begin{abstract}
The appearance of SOA affects business and IT environment and many studies on SOA is progressed in academics and industries. Service will increase extremely and make business opportunity by composing service. In addition, It will support it. However, studies on selection of a service composition target are insufficient. In this study, we propose a framework that select a service composition target so we use Analytic Hierarchy Process methodology. As a result of this study, we enabled service selection to apply functional/non-functional attributes of services, various stakeholder's view and some selection criteria with service composition.
\end{abstract}

Keywords : Service, Composition, Analytic Hierarchy Process

\section{1. 서 론}

현재 세상은 빠르게 변하고 있으며 고객의 요구사항도 복 잡하고 다양해지고 있다. 고객의 요구사항을 빠르게 반영하 기 위해 $\mathrm{SOA}$ (Service Oriented Architecture)에 대한 관심 이 증가하고 있다. 학계나 주요 IT 벤더들에 의해 SOA에 대한 연구 및 구현이 진행되고 있으며, 현재의 객체나 컴포 넌트처럼 서비스도 폭발적으로 증가할 것으로 전망하고 있 다. 고객의 비즈니스 기회를 창출하기 위해 기존 서비스들 은 어떻게(How) 컴포지션하여 새로운 서비스를 생성할 것 인가에 대해 많은 연구가 진행되었다. 그러나 컴포지션의 대상이 되는 서비스(What)를 선정하는 연구는 부족한 실정 이다. 현재 서비스 선정 연구는 서비스 활용의 측면에서 선

\footnotetext{
† 준 회 원 : 숭실대학교 컴퓨터학과 박사과정

†† 준 회 원 : 숭실대학교 컴퓨터학과 석사과정

휘 종신회원 : 숭실대학교 컴퓨터학부 교수

논문접수:2009년 6월 2일

수 정 일 : 1 차 2009년 7월 10일, 2차 2009년 7월 28일

심사완료:2009년 7월 28일
}

정을 연구하거나 서비스 컴포지션의 하위의 요소로써 연구 되었다. 서비스 컴포지션을 바라보는 관점은 다양하며, 서비 스를 선정하는 기준도 매우 다양하기 때문에, 다수 이해관 계자의 다양한 선정기준을 반영할 수 있는 체계적인 선정 방법에 대한 연구가 필요하다.

본 연구는 서비스를 컴포지션 할 때, 의사결정기법 중 하 나인 $\mathrm{AHP}$ (Analytic Hierarchy Process) 방법을 활용하여, 서비스 컴포지션 대상을 선정하는 프레임워크를 제안한다. 선정 프레임워크에서는 서비스 선정 모델과 선정 프로세스 를 포함하고 있다. 그러나 본 연구에서는 선정요소를 식별 하는 것은 연구 범위에서 제외하고 있다.

본 연구를 수행하기 위해, 우선 기존 서비스 조합 모델과 서비스 선정 모델에 대해서 조사 및 분석을 수행한다. 그리 고 $\mathrm{AHP}$ 를 조사하고 이를 활용할 수 있는 방법에 대해 도출 한다. 관련 연구를 기반으로 컴포지션을 위한 대상 서비스 선정 모델과 선정 프로세스를 제안하고 적용 및 검증하여 본 연구에서 제안하는 프레임워크가 유용함을 보인다. 


\section{2. 관련 연구}

본 연구의 목적을 달성하기 위해, 우선 서비스 조합 모델 과 선정 모델을 살펴보고, 본 연구의 기반이 될 $\mathrm{AHP}$ 에 대 해서 살펴볼 것이다.

\section{1 기존 서비스 조합 모델에 관한 연구}

서비스 컴포지션은 크게 수동/자동/반자동 컴포지션으로 구분될 수 있다. 수동 컴포지션은 WSFL(Web Service Flow Language)나 BPEL(Business Process Execution Language) 와 같은 서비스 Flow 명세 언어를 이용하여 컴포지션 되는 서비스를 수동으로 모델화 하는 것이다[1-6]. 그러나 수동 컴포지션 접근법은 사람의 많은 노력이 필요한 단점이 존재 한다.

자동 컴포지션은 서비스 발견 에이전트를 생성하는 연구 이다[7-12]. 그러나 자동 컴포지션의 단점은 컴포지션 이전 에 서비스에 대한 명시적인 조건(서비스 요청자가 컴포지션 의 입력, 출력, 오퍼레이션을 명확하게 알아야만 한다)을 알 아야 하며, 서비스의 수가 증가함에 따라 명시적인 조건에 부합하는 서비스가 다수 존재할 가능성이 있다. 그리고 단 지 서비스 내부의 정보만을 이용하기 때문에 컴포지션 목적 에 불필요한 다수의 조합 결과를 생성할 수 있다는 단점이 있다. 그러므로 조합의 목적 또는 조건이 명시적이면서 동 적일 수 있을 방안이 필요하다.

반자동 컴포지션은 수동 컴포지션과 자동 컴포지션 접근 법을 혼합한 전략이다. 자동 컴포지션을 통해 컴포지션 서 비스 구조의 형태의 대안을 만들고, 사람의 노력으로 대안 을 선택하거나 다시 컴포지션을 시도하는 것이다. 반자동 컴포지션의 구조와 형태에 대한 연구로 자동 컴포지션의 연 구들이 대부분 활용될 수 있으며, 그래프를 이용한 방법[13] 도 존재한다. 반자동 컴포지션의 경우, 요청자의 의견이 선 택을 통해 표현되지만, 선택의 범위가 시스템에 의해 도출 된 대안의 범위에 한정되는 단점이 존재한다. [27]은 비기능 속성 중 과거의 서비스 조합 사례를 기반으로 서비스를 조 합하는 연구이다. 과거 사례 기반이 정확히 일치하지 않는 경우, 유사한 사례를 기반으로 서비스를 조합하는 방법이며, 적용된 기법은 Limited Radius Clustering 방식을 사용하며 유사 사례 선정 기준으로 시간 유사도와 비용 유사도를 이 용하였다.

이러한 서비스 컴포지션 모델은 본 연구에 중요한 기반이 된다. 그러나 본 연구는 어떻게(How)가 아닌 어떠한 서비스 (What)를 컴포지션하는지에 대한 연구이므로 다루는 범위가 다소 다르다.

\section{2 기존 서비스 선정 모델}

서비스 선정에 대한 기존 연구는 기능/오퍼레이션 기준 선 정, 비기능 속성 고려, 측정 및 평가 방법을 연구하고 있다.

기능/오퍼레이션 기준 선정 방법에 대한 연구 중 하나인 $[14,15]$ 는 서비스를 선정하기 위한 온톨로지 모델의 정의와
프레임워크를 제안하였다. 유사한 국내 연구로 [25]가 존재 한다. 그러나 각 연구의 저자는 기능/오퍼레이션을 중심으로 하였기 때문에 비기능적인 속성을 반영하기 힘들을 한계점 으로 밝히고 있다.

비기능 속성을 고려한 서비스 선정 방식으로 [16]은 과거 오퍼레이션 중심의 선정에서 반영하기 힘들었던 비기능 속 성을 고려하고자 서비스 선정에서 고려해야 하는 비기능 속 성을 나열하고 5 개의 항목으로 분류하였다. 그러나 이 연구 는 나열과 분류 이외에 제시한 속성을 활용/측정법을 설명 하지 못하고 있다. [17-19] 연구는 [16]에서 제시한 속성을 기반으로 서비스 선정시 비기능 속성을 고려할 수 있는 QoS 기반 서비스 선택에 대한 연구를 수행하였다. 이 연구 들은 비기능 속성을 고려하기 위한 목적으로 실질적인 측정 및 평가를 수행하고 있으나, 평가의 기준을 제시하지 못한 단점이 있다. 이와 유사한 국내 연구로 [26]이 존재한다. [26]은 비기능 속성 중 서비스의 신뢰성을 기준으로 서비스 를 선택하는 기법을 연구한 논문이다. 이 연구는 기존 비기 능 속성 기반 선정 방법에 비해 구체적인 선정 기준(신뢰도 정보)을 활용한 평가 자료 구조, 개념적 프레임워크를 제안 하였다. 그러나 이 연구 역시 기존 비기능 속성 기반 선정 방법과 마찬가지로 서비스 선정의 방법으로 특정 비기능 속 성(신뢰도)에 국한된 기법을 제안하고 있다.

특정 비기능 속성에 국한되지 않는 연구인 [20]은 서비스 선정시 비기능 속성을 반영할 수 있는 메타 모델을 정의하 고 각 속성 사이의 관계에 LSP(Logic Scoring of Perferences) 를 활용하여 근거를 마련하였다. 그리고 각 속성에 대한 메 트릭을 제안하고 이를 적용하였다. [20]은 서비스 선정에 비 기능 속성을 고려할 때의 가이드라인과 방법을 제안하였으 나 제안한 메타 모델에선 서비스 컴포지션의 요소를 고려하 지 않고 있는 한계점이 존재한다.

\subsection{AHP (Analytic Hierarchy Process)}

AHP는 1980년에 Tomas Saaty에 의해 제안된 다수 대안 에 대한 다면적 평가기준을 통한 의사결정지원 방법이다 [21]. 의사결정을 위한 사항들을 식별하고 계층적으로 분류 하여 의사결정 모형을 설정한다. 의사 결정 모형에서 동일 수준의 사항에 대해서 쌍대비교(pairwise comparison)를 통 해 가중치 판단자료를 수집하고 각 사항의 상대적 가중치를 결정한다. 계층 구조와 기준 및 대안의 상대적 가중치를 종 합하여 의사결정에 활용한다. $\mathrm{AHP}$ 방법은 상대적인 기준으 로 절대적인 수치를 계산할 수 있기 때문에 많은 분야에서 평가 및 결정시 활용되고 있다. 본 연구에서는 $\mathrm{AHP}$ 를 이용 하여 서비스 선정 기준들을 구조화하고 가중치를 계산한다. $\mathrm{AHP}$ 의 프로세스는 본 연구에서 제안하는 서비스 선정 프로 세스의 일부로 포함된다.

\section{3. 서비스 선정 프레임워크의 제안}

서비스 컴포지션 대상 선정 프레임워크를 제안하기 위해, 
우선 선정 프레임워크의 전반적인 개요와 가정을 제시한다. 그리고 서비스 선정 프레임워크를 구성하는 서비스 선정 프 로세스와 서비스 선정 모델을 제안한다.

\section{1 서비스 선정 프레임워크의 가정과 개요}

서비스를 컴포지션 해야 하는 이유는 비즈니스 동인 (Motive)에 의해서 발생하며, 이러한 동인을 Business Goal 이라고 본 연구에서는 정의한다. 본 연구는 (그림 1)과 같이 Business Goal과 이를 달성할 수 있는 후보 서비스들이 식 별되어 있는 상황에서 어떠한 서비스를 선정하여 조합해야 하는지 결정할 수 있는 프레임워크에 대한 연구이다.

연구를 수행하기 위하여 OASIS SOA Reference Model[22] 에서 정의한 서비스의 특징을 기반으로 다음과 같은 가정을 도출하였다.

[가정 1] 서비스는 하나 이상의 기능 속성(Functionality) 를 표현한다.

[가정 2] 동일한 기능 속성을 표현하는 서비스는 무한히 많다. 그러나 이들은 비기능 속성(Non-Functionality)에 의 해 구분될 수 있다.

[가정 3] 모든 서비스는 컴포지션 가능하다. 그러나 투입 되는 자원 및 획득되는 가치는 비기능 속성에 의해 달라진다.

본 연구는 Non-Functionality한 요소가 무엇인지 밝히는 연구는 아니지만 [16]에서 도출한 5가지 분류의 비기능 속성 을 반영할 수 있다. 가정 3 은 서비스의 특징 중 하나이고 본 연구에서는 추가로 컴포지션을 수행하기 위한 노력이 서 비스의 비기능 속성에 따라 달라진다고 가정하고 있다.
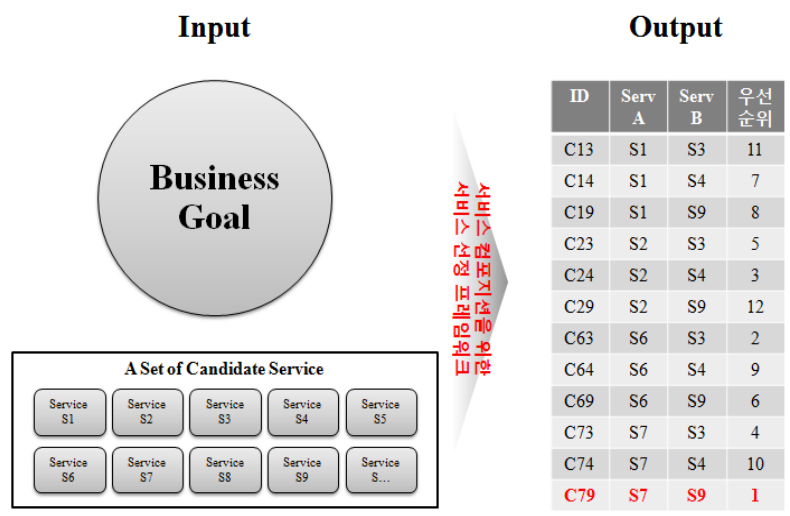

(그림 1) 컴포지션을 위한 서비스 선정 프레임워크의 전재조건 과 목표

\section{2 서비스 선정 프로세스}

컴포지션의 대상이 되는 서비스를 선정하기 위해, 우선 주어진 Business Goal을 분석하여 조합해야할 기능 속성들 을 식별한다. Business Goal을 분석하여 기능 속성의 집합 을 도출하는 방법에 대한 방법들이 존재한다. 이러한 방법 을 활용하여 Business Goal에서 기능 속성을 도출한다.

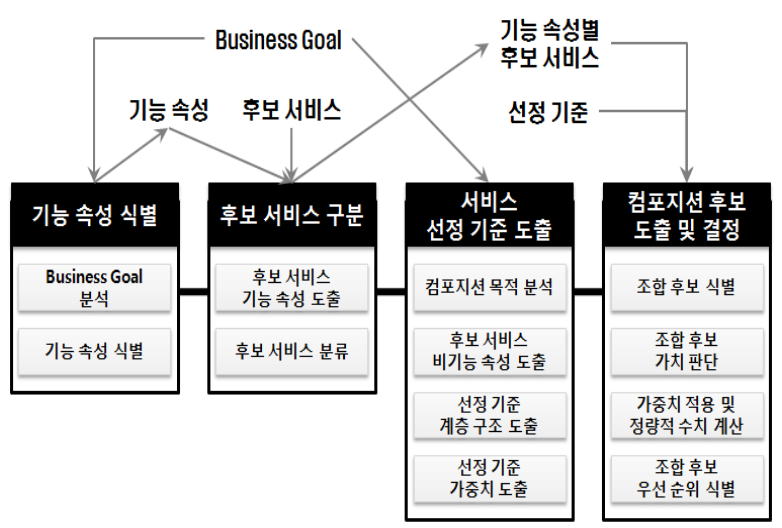

(그림 2) 서비스 컴포지션 대상 선정 프로세스

두 번째 단계는 도출한 기능 속성을 기준으로 후보 서비 스 집합을 구분한다. 만약 어떠한 기능 속성에도 포함되지 않는 경우는 제거하여 후보 서비스의 수를 줄일 수 있으며, 동시에 여러 기능 속성을 가질 경우, 각 기능 속성 모두에 포함하면 된다.

세 번째 단계는 목적을 달성할 때의 중요 고려사항이 무 엇인지 식별하여 컴포지션 서비스 선정 기준을 도출한다. 선정 기준은 서비스의 비기능 속성을 고려해야만 한다. 선 정 기능의 예시로는 컴포지션 비용, 획득 이득, 서비스 유지 비용, 리스크, 복잡도 등 여러 가지가 될 수 있다. 고려 가능 한 요소로는 [16] 연구의 비기능 속성 요소를 이용할 수 있 다. 이러한 선정 기준을 가지고 $\mathrm{AHP}$ 기법을 활용하여 계층 구조 및 각 선정 기준별 가중치를 결정한다.

네 번째 단계는 각 기능 별로 조합 가능한 조합 후보들을 식별한다. 그리고 조합 후보별로 각 선정 기준에 대한 판단 을 한다. 그리고 그 결과에 가중치를 고려하여 정량적인 결 과 수치를 도출한다. 결과 수치를 기준으로 우선순위를 식 별하는 것으로 서비스 선정 프로세스를 종료한다. (그림 2) 는 본 연구에서 제안하는 서비스 선정 프로세스의 각 단계 및 활동과 입출력 요소를 표현한 것이다.

\section{3 서비스 선정 모델}

서비스 선정 모델은 서비스 선정 프로세스의 단계별 관점 이 아닌 입출력 관계를 중점으로 도출한다. 우선 Business $\mathrm{Goal}$ 을 실체화한 것이 시스템이며, 시스템은 다수의 서비스 로 구성되어 있다. 각 서비스는 하나 이상의 기능 속성을 표현하고 있다. 결과적으로 Business Goal을 기반으로 기능 속성을 표현할 수 있다. (그림 3-a)는 Business Goal과 기능 속성 사이의 관계를 표현한 것이다. 이를 기반으로 (그림 3-b)와 같이 Business Goal을 기반으로 기능 속성 F1, F2 (혹은 그 이상)를 식별할 수 있다.

식별한 기능 속성별로 후보 서비스 집합을 분류할 수 있 다. 이는 기능 속성별 후보 서비스 구분 단계에서 수행되는 과정이다. (그림 4-a)는 기능 속성, 후보 서비스 집합, 후보 서비스 사이의 관계를 표현한 것이다. 이를 기반으로 (그림 4-b)와 같이 F1에 속하는 서비스 집합(S1, S2, S6, S6)과 


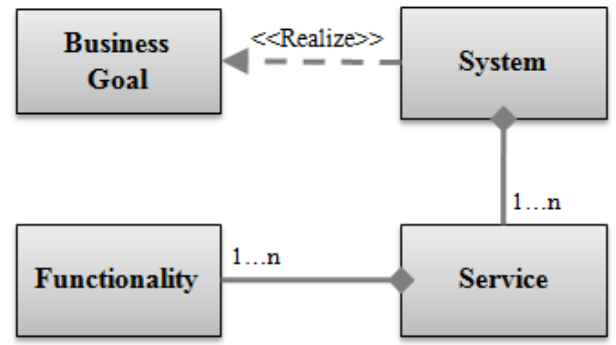

(a)

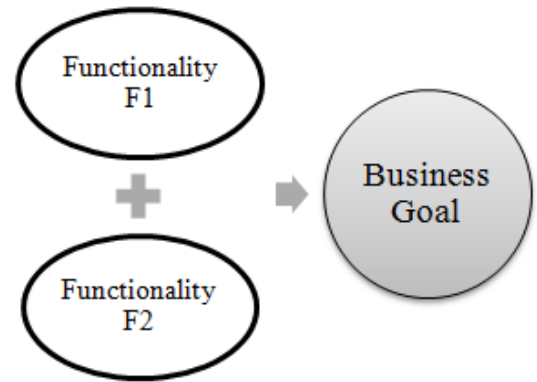

(b)

(그림 3) Business Goal과 기능 속성 사이의 관계

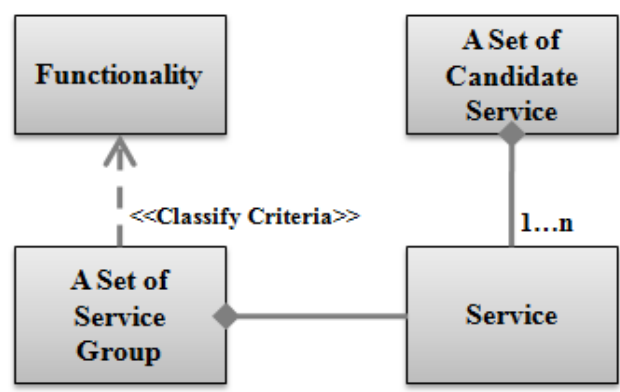

(a)

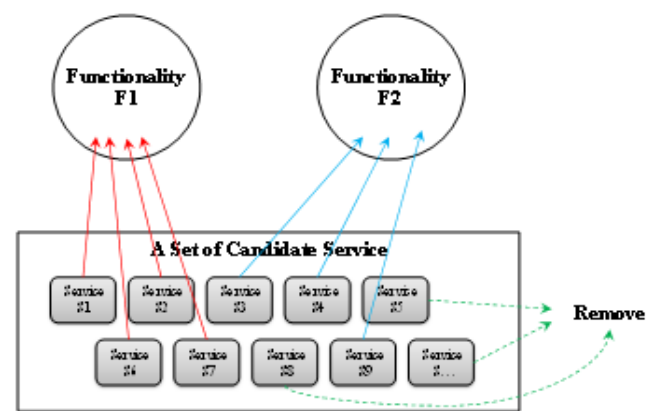

(b)

(그림 4) 기능 속성, 후보 서비스 집합, 후보 서비스 사이의 관계

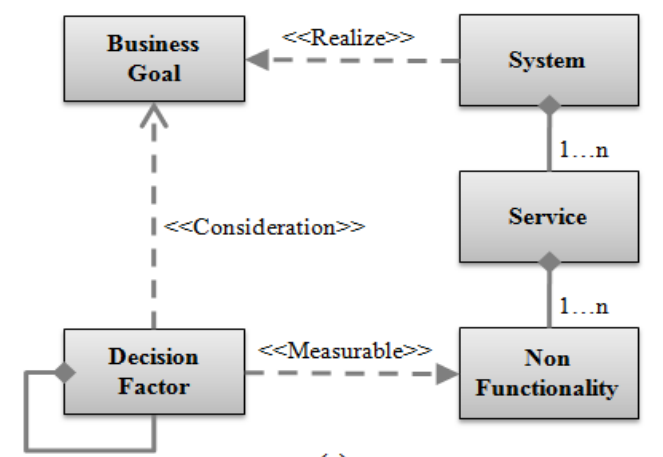

(a)

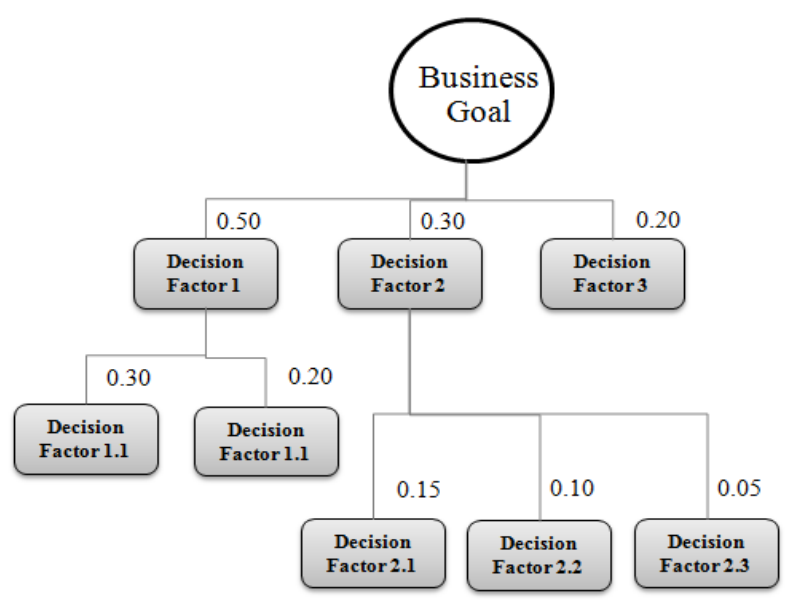

(b)

(그림 5) Business Goal과 선정 기준 사이의 관계

$\mathrm{F} 2$ 에 속하는 서비스 집합 $(\mathrm{S} 3, \mathrm{~S} 4, \mathrm{~S} 9)$ 그리고 제거되는 후 보 서비스를 찾을 수 있다.

서비스 선정을 위하여 선정 기준을 식별해야 한다. 이는 Business Goal을 고려하고 각 서비스의 비기능 속성을 고려 하여, 정량적 또는 정성적으로 측정 가능한 선정 기준 (Decision Factor, DF)을 구분한다. 이는 $\mathrm{AHP}$ 기법을 활용 하여 (그림 5-b)와 같이 계층구조를 이루고, 동등한 수준의 선정 기준에 대한 쌍대비교(Pairwise Comparision)를 통해 $<$ 표 $1>$ 과 같이 선정 기준별 가중치를 구할 수 있다. 2 개의 임의의 선정 기준 사이의 주관적인 중요성 평가를 객관적인
가중치로 변경할 때, 쌍대비교가 사용된다. <표 2>는 $\mathrm{AHP}$ 〈표 1〉도출된 선정기준과 가중치

\begin{tabular}{|c|c|c|}
\hline \multicolumn{2}{|c|}{ Decision Factor } & Weight \\
\hline \hline DF1 & DF1.1 & 0.30 \\
\hline & DF1.2 & 0.20 \\
\hline DF2 & DF2.1 & 0.15 \\
\hline & DF2.2 & 0.10 \\
\hline \multicolumn{2}{|c|}{ DF3 } & 0.05 \\
\hline & DF2.3 & 0.20 \\
\hline
\end{tabular}


〈표 2〉가중치 결정을 위한 기본 척도 ${ }^{[21]}$

\begin{tabular}{|c|c|c|}
\hline $\begin{array}{c}\text { 중요성 강도 } \\
\text { (Intensity of } \\
\text { Importance) }\end{array}$ & $\begin{array}{c}\text { 정의 } \\
\text { (Definition) }\end{array}$ & $\begin{array}{c}\text { 설명 } \\
\text { (Explanation) }\end{array}$ \\
\hline \hline 1 & Equal & $\begin{array}{c}\text { 2개 요소가 목적에 미치는 영향이 } \\
\text { 비슷함 }\end{array}$ \\
\hline 3 & Moderate & $\begin{array}{c}1 \text { 개 요소가 경험과 판단을 기준으로 } \\
\text { 약간 중요함 }\end{array}$ \\
\hline 5 & Strong & $\begin{array}{c}1 \text { 개의 요소가 경험과 판단을 기준으로 } \\
\text { 확실하게 중요함 }\end{array}$ \\
\hline 7 & Very Strong & $\begin{array}{c}1 \text { 개의 요소가 경험과 판단을 기준으로 } \\
\text { 매우 중요함 }\end{array}$ \\
\hline 9 & Extreme & $\begin{array}{c}1 \text { 개의 요소가 매우 중요하다는 } \\
\text { 겅적인 증거가 있거나 우선순위의 } \\
\text { 차이가 매우 확실함 }\end{array}$ \\
\hline
\end{tabular}

에서 널리 사용되는 Satty의 기본 척도 [21]을 표현한 것이 다. (그림 5-a)는 Business Goal과 선정 기준 사이의 관계를 표현한 것이며, 이를 기반으로 (그림 5-b)와 같이 선정 기준 (DF1.1, DF1.2, DF2.1, DF2.2, DF2.3, DF3)을 및 가중치(계 산의 편의성을 위해 $\mathrm{AHP}$ 의 룰을 벗어나지 않도록 임의의 수치로 정하였음)를 식별할 수 있다.

기능 속성별로 구분한 서비스 집합의 조합을 통해 조합 가능한 조합 후보를 식별한다. 각 조합 가능한 서비스 후보 별로 도출한 선정 기준에 따라 평가를 수행한다. 그리고 그 평가 결과와 선정 기준별 가중치를 곱하고 모든 선정 기준 별 결과를 합산하여 계산 결과를 만든다. <표 $3>$ 은 기능별

〈표 3〉 조합 후보별 선정 기준에 따른 계산과 우선순위

\begin{tabular}{|c|c|c|c|c|c|c|c|c|c|c|}
\hline ID & F1 & $\mathrm{F} 2$ & $\begin{array}{l}\text { DF1.1 } \\
(\mathrm{x} 0.30)\end{array}$ & $\begin{array}{c}\mathrm{DF} 1.2 \\
(\mathrm{x} 0.20)\end{array}$ & $\begin{array}{l}\text { DF2.1 } \\
(\mathrm{x} 0.15)\end{array}$ & $\begin{array}{l}\mathrm{DF} 2.2 \\
(\mathrm{x} 0.10)\end{array}$ & $\begin{array}{l}\text { DF2.3 } \\
(\mathrm{x} 0.15)\end{array}$ & $\begin{array}{r}\text { DF3 } \\
(\mathrm{x} 0.20\end{array}$ & $\begin{array}{l}\text { 계산 } \\
\text { 결과 }\end{array}$ & $\begin{array}{l}\text { 우선 } \\
\text { 순위 }\end{array}$ \\
\hline $\mathrm{C} 13$ & S1 & S3 & 1 & 4 & 3 & 2 & 5 & 1 & 2.20 & 11 \\
\hline C14 & S1 & S4 & 3 & 3 & 2 & 4 & 5 & 2 & 2.85 & 7 \\
\hline C19 & S1 & S9 & 4 & 2 & 5 & 1 & 2 & 1 & 2.75 & 8 \\
\hline $\mathrm{C} 23$ & S2 & S3 & 5 & 1 & 3 & 4 & 2 & 3 & 3.25 & 5 \\
\hline C24 & S2 & S4 & 2 & 5 & 4 & 4 & 3 & 3 & 3.35 & 3 \\
\hline C29 & S2 & S9 & 1 & 3 & 4 & 2 & 2 & 2 & 2.20 & 12 \\
\hline C63 & S6 & S3 & 3 & 4 & 3 & 3 & 3 & 4 & 3.40 & 2 \\
\hline C64 & S6 & S4 & 2 & 2 & 2 & 3 & 4 & 3 & 2.40 & 9 \\
\hline C69 & S6 & S9 & 4 & 2 & 2 & 4 & 3 & 3 & 3.05 & 6 \\
\hline C73 & S7 & S3 & 5 & 3 & 3 & 2 & 3 & 2 & 3.30 & 4 \\
\hline C74 & S7 & S4 & 2 & 3 & 4 & 1 & 2 & 1 & 2.20 & 10 \\
\hline C79 & S7 & S9 & 3 & 4 & 1 & 5 & 2 & 5 & 3.45 & 1 \\
\hline
\end{tabular}

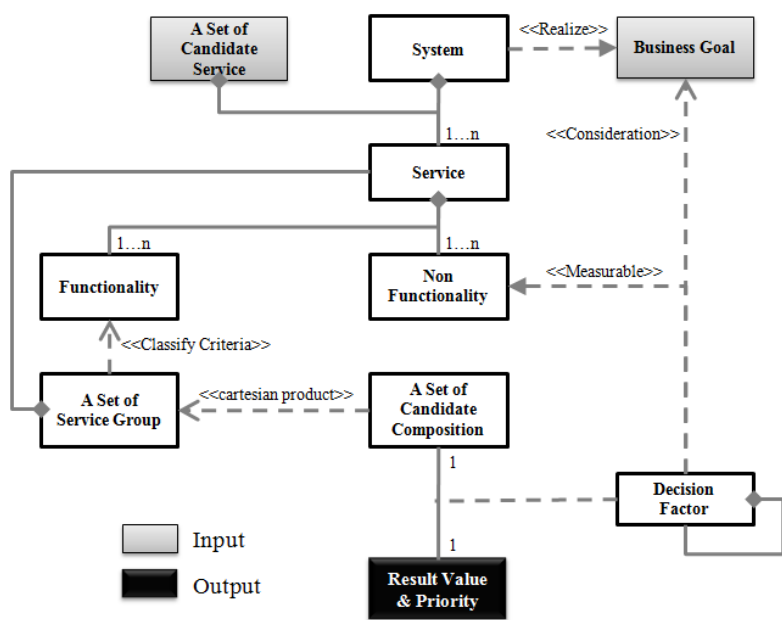

(그림 6) 서비스 컴포지션 대상 선정 모델

집합별 컴포지션 가능한(Cartesian product) 조합 후보 12개 를 식별하고 각 선정 기준별로 Likert 5점 척도[24]를 사용 한 예시이다. 각 선정 기준의 가중치는 괄호에 표현하였으 며, 5점 척도는 1점이 매우 나쁨에서 5점이 매우 좋음으로 나타냈다. 총 계산 결과의 범위는 1 5까지이며, 5 에 가까울 수록 조합이 비즈니스 목적에 적합하고 선정 기준에 가장 적합한 서비스 조합으로 볼 수 있다. 조사 결과에 가중치를 적용하여 계산하고 이를 기반으로 우선순위를 결정한다.

<표 3>에 따르면 서비스 S7과 서비스 S9의 컴포지션(ID : C79)이 가장 높은 점수를 가짐으로 높은 우선순위를 가지 게 된다. 서비스 선정 모델의 입출력과 가정을 기반으로 도 출한 모델들을 (그림 6)과 같이 종합하였다.

Business Goal을 실현하기 위하여 후보 서비스 중 일부 를 선정하여 조합함으로써 시스템을 구축한다. 서비스는 하 나 이상의 기능 속성과 비기능 속성을 가진다. 기능 요소를 기반으로 서비스를 그룹으로 분류하고, 분류된 서비스를 이 용하여 조합 후보를 선정한다. 서비스의 비기능 요소는 비 즈니스 목적을 고려하여 비기능 요소를 측정할 수 있는 선 정 기준을 수립한다. 선정 기준을 고려하여 조합 후보별 Value를 계산하고 이를 기준으로 우선순위를 결정한다.

\section{4. 검 증}

본 연구의 검증을 위해 관련 연구에서 설명한 기존 서비 스 선정 모델과 제안한 연구의 비교와 비교 결과를 뒷받침 할 수 있는 사례 연구를 수행하였다.

\section{1 기존 서비스 선정 모델과의 비교}

본 연구와 동일한 범위를 가지는 연구가 기존에 미흡한 실정이다. 그러나 본 연구와 유사한 기존의 연구와 비교하 여 본 연구의 차별성을 부각하고자 한다. 비교 결과는 <표 $4>$ 와 같다. 선정 방법 항목은 관련 연구에서 조사한 기능 속성 기반 서비스 선정 연구 $[14,15,25]$ 와, 비기능 속성을 
〈표 4〉기존 선정 방법과 제안한 선정 방법의 비교

\begin{tabular}{|c|c|c|c|c|c|}
\hline 선정 방법 & 기능 속성 고려 & 비기능 속성 고려 & 비기능 속성 선정 기준의 정의 & 다수 기준 적용 & 컴포지션 결과 고려 \\
\hline \hline 기능 기반 선정 & $\mathrm{O}$ & $\mathrm{X}$ & $\mathrm{X}$ & $\mathrm{X}$ & $\mathrm{O}$ \\
\hline 비기능 기반 선정 & $\mathrm{O}$ & $\mathrm{O}$ & $\mathrm{X}$ & $\mathrm{X}$ & $\mathrm{O}$ \\
\hline 메타 모델 기반 선정 & $\mathrm{X}$ & $\mathrm{O}$ & $\mathrm{O}$ & $\mathrm{O}$ & $\mathrm{X}$ \\
\hline 제안한 방법 & $\mathrm{O}$ & $\mathrm{O}$ & $\mathrm{O}$ & $\mathrm{O}$ & $\mathrm{O}$ \\
\hline
\end{tabular}

고려한 방법인 [17-19,26], 비기능 속성을 고려한 메타모델 기반 방법[20]을 비교 대상으로 선정하였다.

기능 속성 고려 항목은 서비스 선정시 서비스의 기능 (오 퍼레이션, 입력, 출력, 사전조건, 사후조건 등)을 고려할 수 있는지 여부를 표현한 것이다. 기능 기반 선정 방법의 경우 서비스 오퍼레이션의 각 정보(입력, 출력, 등)을 저장하고 이를 활용할 수 있다. 반면 메타 모델 기반 선정 방법 연구 는 서비스 선정 메타 모델에서 기능 속성에 대한 언급이 없 었다.

비기능 속성 고려 항목은 서비스 선정시 비기능 속성에 대해 고려하고 있는지 여부를 표현한 것이다. 기능 속성 기 반 선정 방법의 경우, 온톨로지 기반의 초기 서비스 선정 연구로써 비기능 속성을 고려하고 있지 못함을 연구의 한계 점으로 밝히고 있다. 비기능 속성 기반 선정 방법은 기능 속성 기반 선정 방법의 한계점을 개선한 연구이다.

비기능 속성 선정 기준 정의 항목은 서비스 선정시 고려 할 비기능 속성을 선정자가 임의로 정할 수 있는가에 대한 여부이다. 다수 기준 적용 항목 역시 서비스 선정시 다수의 기준을 적용할 수 있는가에 대해 표현한 것이다. 온톨로지 기반 방법의 경우 도메인 온톨로지를 필요로 하고, 서비스 선정에 필요한 선정 엔진(추론/선출 엔진 등)을 필요로 한 다. 그리고 온톨로지를 그래프로 표현하기 위한 방법이 필 요하다. [26]의 경우 온톨로지를 사용하지 않은 기능/비기능 속성을 고려한 선정 방법이다. 그러나 [26]은 비기능 속성 기반 선정 방법과 비슷하게 특정 비기능 속성(신뢰성)에 한 정된 프레임워크를 제안하고 있다. 종합적으로 비기능 속성 기반 선정 방법의 경우, 임의의 비기능 속성을 기준으로 서 비스를 선정할 수 없는 한계가 존재한다. 반면 메타 모델 기반 선정 연구는 비기능 속성을 임의로 선정하고 다수 기 준을 적용할 수 있는 메타 모델을 제안하고 있다. 그러나 구체적인 방법을 제안하고 있지 않아 서비스 조합 결과를 예측할 수 없는 단점이 있다.

컴포지션 결과 고려 항목은 서비스 선정 결과를 고려할 수 있는지 여부에 대한 질문이다. 기능 속성 기반 및 기능/ 비기능 속성 기반 서비스 선정 연구는 컴포지션 결과를 고 려할 수 있는 장점이 있다. 이는 서비스 오퍼레이션에 대한 정보를 도메인 온톨로지가 담고 있기 때문이다. 반면 [20] 연구는 메타 모델에서 컴포지션 요소를 고려하지 않고 있지 때문에 서비스 조합의 결과를 예측할 수 없다.

제안한 방법과 대조군(기능 속성 기반 선정 방법, 비기능 기반 선정 방법, 비기능 메타모델 기반 선정 방법)에 대해
각 항목의 조사 결과를 정리한 것이 <표 $4>$ 이다. 제안한 방 법은 기존 연구의 다음과 같은 장점들을 수용하였다.

첫째, 본 연구는 서비스의 기능성과 비기능성을 모두 고 려하고 있다. 본 연구에서 제안하는 절차와 메타모델을 보 면 서비스를 후보 서비스로 분류하는 과정은 기능 속성을 고려하고 있다. 기능적으로 올바르게 조합되지 않는 서비스 를 미리 고려한다는 의미이다.

둘째, 본 연구는 비기능 속성을 고려하고 있다. 기능적으 로 올바른 후보 서비스 중 최선의 서비스를 결정하는 것은 비기능 속성에 의해 결정된다.

셋째, 본 연구는 비기능 속성 선정 기준을 선정자가 정의 할 수 있다. 그리고 본 연구는 다수의 기준 적용이 가능하 다. 그리고 본 연구는 특정한 물리적 시스템이 존재하지 않 아도 사람이 직접 수작업으로 선정할 수 있도록 제안하였 다. 또한 다수의 선정 기준을 정의하고 적용할 수 있게 $\mathrm{AHP}$ 방법을 활용하여 이를 반영할 수 있게 하였다.

마지막으로 본 연구는 컴포지션 결과 고려할 수 있다. 컴 포지션 결과는 도출하기 위해 비즈니스 목표에 적합한 기능 속성을 고려하여 서비스를 분류하고 후보 서비스를 도출하 며 도출한 후보 서비스에 대한 비기능 속성을 고려하여 최 종 서비스를 선정한다. 본 연구에서 제안한 (그림 2)의 절차 에 따라 서비스 선정 모델과 같이 컴포지션 결과를 예측할 수 있다.

\section{2 사례 연구}

본 사례 연구는 $\mathrm{N}$ 게임사의 온라인 게임의 지형 제작 도 구를 .NET Framework 3.5의 WCF를 적용하여 서비스화한 사례의 일부분이다. 서비스는 총 4 개의 서비스 서버에 등록 되어 있으며 서비스 서버는 IIS 6.0을 사용하였다. <표 5>

〈표 5〉지형 제작 도구를 구성하는 서비스 목록

\begin{tabular}{|c|c|}
\hline 서비스 명 & 유사 서비스 (XXXX) \\
\hline \hline Terrain_Presentation_XXXX & 1073 \\
\hline Terrain_Func_Interface_XXXX & 1073 \\
\hline Terrain_Business_Proc_XXXX & 1059,1073 \\
\hline Terrain_Composition_Serv_XXXX & 1059,1073 \\
\hline Terrain_TileSet_Serv_XXXX & 1059,1073 \\
\hline Terrain_Object_Serv_XXXX & 1059 \\
\hline Terrain_HighLow_Serv_XXXX & 1032,1059 \\
\hline Terrain_DB_Serv_XXXX & $0976,1031,1032$ \\
\hline
\end{tabular}




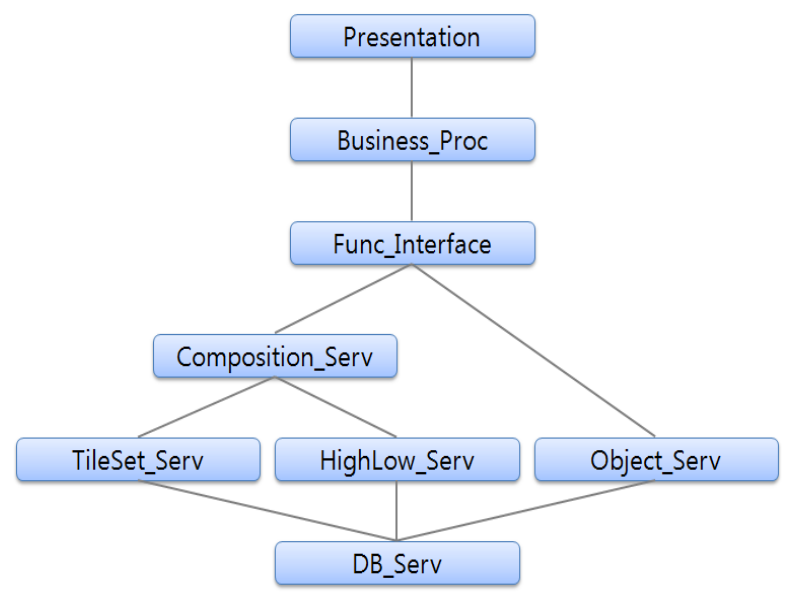

(그림 7) 서비스 조합 트리

는 지형 제작이라는 Business Goal을 기반으로 수집한 서비 스 이다. 서비스 명 항목은 지형 제작 도구를 구성하기 위 해 필요한 서비스들 $(\mathrm{XXXX}$ 는 각 서비스의 버전을 의미)이 다. 유사 서비스 항목은 각 서비스의 배포 버전을 표기한 것으로 예를 들어 1032의 경우 1.0.3.2를 의미한다. 각 버전 의 차이는 인터페이스는 동일하나 내부 구현 방법에 따라 각 서비스의 품질 (응답 속도, 에러율 등)이 다르다고 가정 한다. <표 $5>$ 는 제안한 연구의 단계 1 과 2 를 수행하여 서비 스 목록이 도출된 결과이다.

지형 제작 도구를 구성하기 위한 서비스 조합 트리는 (그 림 7)과 같다. <표 4>의 비교와 같이 기능 속성 기반 선정 방법의 경우, 인터페이스만 비교하기 때문에, 가능한 결과의 수는 48 가지가 된다. 모든 버전은 인터페이스가 동일하기 때문에 서비스 오퍼레이션 비교에 의한 결과는 너무 많은 종류의 조합 경우를 생성하게 된다.

비기능 속성 기반 선정 방법의 경우, 기능 속성 기반 선 정 방법에 비기능 속성을 고려하는 방법이다. 그러나 (그림 8)에서와 같이 $\mathrm{AHP}$ 를 사용하여 선택된 특정 기준 (응답시 간이 $130 \mathrm{~ms}$ 이하)에 적합한 경우, 해당하는 서비스가 다수 존재함을 알 수 있었다. 또한 Composition_Serv의 서비스 조합 대상 선정과 TileSet_Serv의 서비스 조합 대상 선정과 같이 서로 다른 서비스에서 모든 서비스 선정에 동일하게

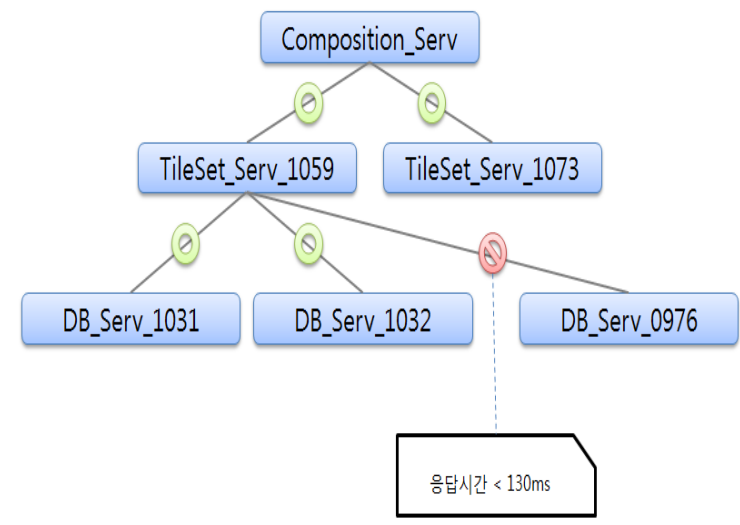

(그림 8) 비기능 속성 기반 서비스 선정 결과 (일부)

정해진 조건을 적용하는 문제점도 발생하였다. [26]의 경우, 선정자가 선택한 서비스 선정 기준이 신뢰성이 아님으로 적 용할 수 없는 문제점도 발생하였다.

단계 1,2 에 따라 도출된 필요 기능과 후보 서비스 <표 $5>$ 를 식별한다. 제안한 방법의 단계 3에 따라, 지형 제작 도 구의 목적에 부합한 여러 가지 비기능 속성 중 서비스 선정 을 위한 비기능 속성 기준은 응답시간, 가용비율, 소모 자원 (소모 $\mathrm{CPU}$, 소모 $\mathrm{RAM}$ )으로 식별하였다. 식별된 선정 기준 을 $\mathrm{AHP}$ 방법을 활용하여 계층구조 도출하고 가중치(0.50, $0.30,0.20$ )를 계산하였다 (그림 9). 단계 4에 따라 식별된 선 정 기준과 가중치를 각 서비스 후보 조합에 적용한 결과는 <표 6>과 (그림 10)과 같다.

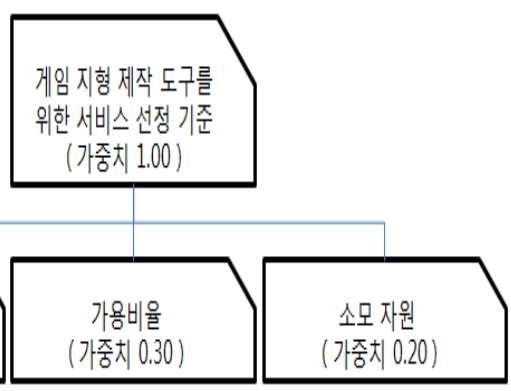

(그림 9) 선정 기준의 계층구조와 가중치

〈표 6〉조합 후보별 선정 기준 가중치 적용 결과 (일부)

\begin{tabular}{|c|c|c|c|c|c|c|c|c|}
\hline Group & ID & 서비스 1 & 서비스 2 & $\begin{array}{l}\text { 응답시간 } \\
(\mathrm{x} 0.50)\end{array}$ & $\begin{array}{c}\text { 가용비율 } \\
\text { (x0.30) }\end{array}$ & $\begin{array}{l}\text { 소모자원 } \\
\text { (x0.20) }\end{array}$ & $\begin{array}{l}\text { 계산 } \\
\text { 결과 }\end{array}$ & $\begin{array}{l}\text { 우선 } \\
\text { 순위 }\end{array}$ \\
\hline \multirow{2}{*}{$\begin{array}{l}\text { Comp }^{+} \\
\text {TileSet }\end{array}$} & T1059 & Composition_Serv_1073 & TileSet_Serv_1059 & 2 & 4 & 1 & 2.4 & 2 \\
\hline & T1073 & Composition_Serv_1073 & TileSet_Serv_1073 & 3 & 3 & 5 & 3.4 & 1 \\
\hline \multirow{3}{*}{$\begin{array}{c}\text { TileSet } \\
+\mathrm{DB}\end{array}$} & D1031 & TileSet_Serv_1073 & DB_Serv_1031 & 4 & 1 & 2 & 2.7 & 3 \\
\hline & D1032 & TileSet_Serv_1073 & DB_Serv_1032 & 3 & 4 & 3 & 3.3 & 1 \\
\hline & D0976 & TileSet_Serv_1073 & DB_Serv_0976 & 1 & 5 & 5 & 3.0 & 2 \\
\hline
\end{tabular}




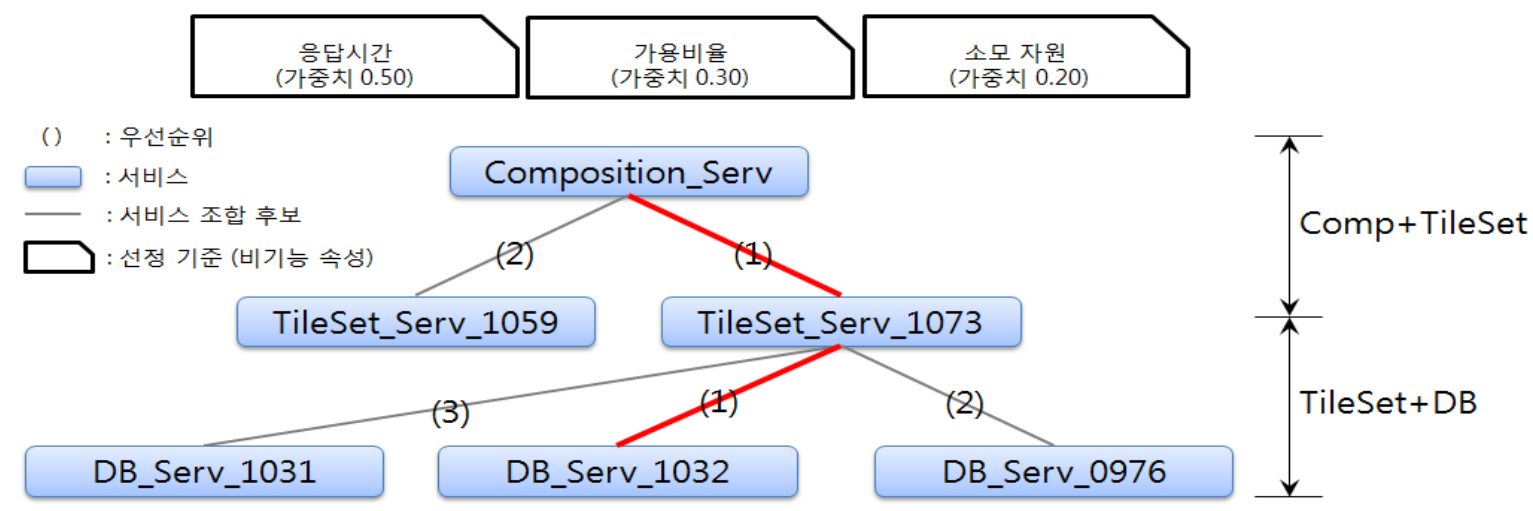

(그림 10) 제안한 연구의 서비스 선정 결과 (일부)

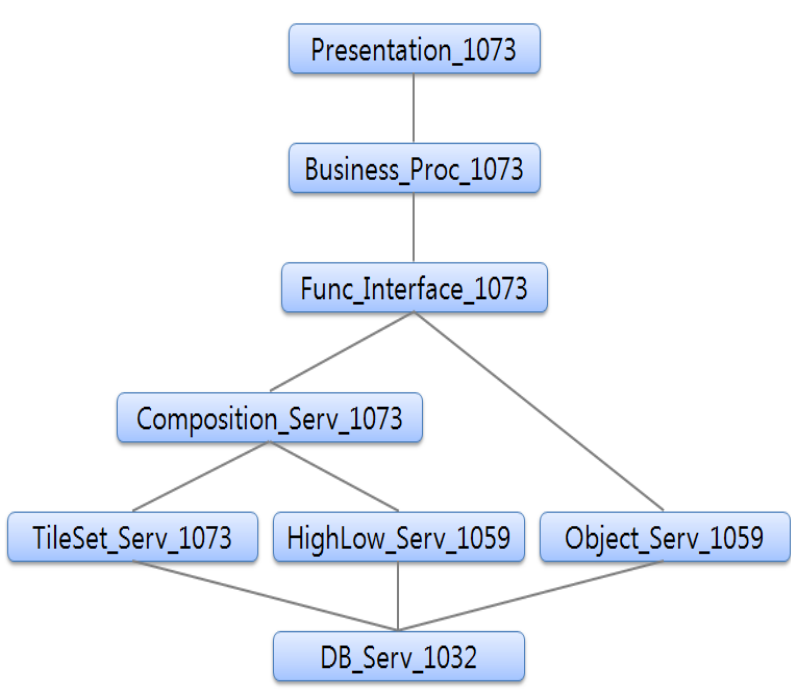

(그림 11) 제안한 연구의 최종 서비스 선정 결과

본 연구에서 제안한 방법을 활용한 최종 선정 결과는 (그 림 11)과 같다. [20]의 경우, <표 $4>$ 컴포지션 결과 고려 항 목에서 보는 바와 같이 다음과 같은 서비스 선정 트리를 생 성하지 못함으로 사례연구에서 제외하였다.

\section{5. 결 론}

본 연구는 컴포지션 하기 위한 서비스를 선정하는 연구이 다. 본 연구는 서비스를 컴포지션 하는 방법(How)에 대한 연구가 아닌 컴포지션 대상을 선정(What)하는 연구이다. 본 연구는 서비스를 선정하기 위해 우선 비즈니스 목표와 서비 스들은 이미 존재하고 있다고 가정하며, 선정한 서비스들은 다양한 방법(Wrapper, Adapter 등)을 활용하여 컴포지션 할 수 있다고 가정하였다.

본 연구는 컴포지션 대상 서비스를 선정하기 위해 우선 비즈니스 목표를 기반으로 시작하였다. 비즈니스 목표에서 도출한 기능 속성을 기반으로 후보 서비스들을 도출한다. 그리고 후보 서비스에서 최종 서비스로 선정하기 위해 비기
능 속성을 고려한다. 다양한 선정 기준(비기능 속성)에 대한 가중치를 $\mathrm{AHP}$ 를 적용하여 도출하고 서비스의 가능한 조합 별로 각 선정 기준과 가중치를 활용하였다. $\mathrm{AHP}$ 를 활용함 으로써 다양한 비기능 속성을 선정자가 직접 정의하여 평가 에 반영할 수 있게 되었다. 본 연구는 선정 절차 (그림 2)를 연구하면서 각 단계의 입/출력 정보를 정리한 서비스 컴포 지션 대상 선정을 위한 메타 모델 (그림 6)도 제안하였다.

제안한 방법은 Satty가 제안한 $\mathrm{AHP}$ 를 활용하여 서비스 조합 대상을 선정하는 방법에 대한 연구이다. $\mathrm{AHP}$ 는 본 연 구에서 비기능 속성을 고려하기 위해 사용되었다. 결과적으 로 본 연구가 제안하는 방법은 반자동 방법과 유사하다고 할 수 있다. 시스템에 의해 제공된 기능적으로 조합 가능한 후보군에 대해 사용자가 선정 기준을 제공하고, $\mathrm{AHP}$ 를 활 용하여 우선순위를 판단하기 때문이다. 그러므로 본 연구는 기존 방식에 비해 다음과 같은 기여가 있다고 고려된다. 첫 째, 서비스 조합의 반자동 선정을 위한 구체적인 방법(그림 2)을 제시하였다. 둘째, $\mathrm{AHP}$ 의 활용으로 인해, 선정시 사용 자에 의한 선정 기준을 동적으로 선택하고 이를 우선순위에 반영할 수 있다. 선정 기준 선택에 대한 메타 모델은 (그림 6)와 같다.

본 연구의 한계는 우선 검증의 범위가 한정되었다는 것이 다. 그리고 각 선정 요소에 대한 통일적인 메트릭을 제안하 지 못했다는 점이 있다. 둘째, $\mathrm{AHP}$ 의 문제로 제시된 Rank Reversal 문제[23]가 본 연구에도 남아 있다는 점이다.

향후 연구로는 본 연구를 기반으로 자동화된 툴을 개발하 고 이를 적용하는 연구이다.

\section{참 고 문 헌}

[1] Casati, F., Ilnicki, S., Jin, L., Krishnamoorthy, V. and Shan, M., "eFlow: A platform for developing and managing composite eServices," HP Laboratories, 2000.

[2] Casati, F., Ilnicki, S., Jin, L., Krishnamoorthy, V. and Shan, M., "Adaptive and dynamic service composition in eFlow," HP Laboratories, 2000. 
[3] Kiwata, K., Nakano, A., Yura, S., Uchihashi, T. and Kanai A., "Scenario-based service composition method in the open service environment," In Proceedings of the Fifth International Symposium on Autonomous Decentralized Systems, 2001.

[4] Tut M. and Edmond, D., "The use of patterns in service composition," In Proceedings of the Workshop on Web Services, e-Business, and the Semantic Web, pp.28-40, 2002.

[5] Aggarwal, R., Verma, K., Miller, J. and Milnor, W., "Constraint driven Web Service composition in METEOR-S," In Proceedings of the IEEE International Conference on Services Computing, pp.23-30, 2004.

[6] Fontoura, M., Lehman, T., Nelson, D., Truong, T. and Xiong, Y. T., "Spaces services suite: Automating the development and management of Web Services," In Proceedings of the 12th International World Wide Web Conference, 2003.

[7] Ponnekanti, S.R., and Fox, A., "SWORD: A developer toolkit for building composite Web Services," In Proceedings of the 11th World Wide Web Conference (Web Engineering Track), 2002.

[8] Thakkar, S., Knoblock, C.A. and Ambite, J.L., "A view integration approach to dynamic composition of Web Services," In Proceedings of the ICAPS Workshop on Planning for Web Services, 2003.

[9] McIlraith, S. and Son, T.C., "Adapting golog for composition of semantic Web Services," In Proceedings of the Eighth Conference on Knowledge Representation and Reasoning, 2002.

[10] Berardi, D., Calvanese, D., Giacomo, G. and Mecella, M., "Reasoning about actions for e-service composition," In Proceedings of the Eighth Conference on Knowledge Representation and Reasoning, 2002.

[11] Wu, D., Sirin, E., Hendler, J., Nau, D. and Parsia, B., "Automatic Web Services composition using SHOP2," In Proceedings of the ICAPS 2003 Workshop on Planning for Web Services, 2003.

[12] Arpinar, B., Aleman-Meza, B., Zhang, R. and Maduko, A., "Ontology-driven Web Services composition platform," In Proceedings of the IEEE International Conference on E-Commerce Technology, pp.146-152, 2004

[13] Qianhui Althea Lang, "AND/OR Graph and Search Algorithm for Discovering Composite Web Services," International Journal of Web Services Research, Vol.2, No.4, pp.46-64, 2005.

[14] I.V. Papaioannou, D.T. Tsesmetzis, I.G. Roussaki, and E.A. Miltiades, "A QoS Ontology Language for Web-Services," AINA'06, Vol.1, pp.101-106, 2006.

[15] D.T. Tsesmetzis, I.G. Roussaki, I.V. Papaioannou and M.E. Anagnostou, "QoS awareness support in Web-Service semantics," AICT-ICIW'06, pp.128, 2006.

[16] S.P. Ran, "A Model for Web Services Discovery with QoS," ACM SIGecom Exchanges, Vol.4, No.1, pp.1-10, 2003.

[17] Y. Liu, A.H.H. Ngu and L. Zeng, "QoS Computation and Policing in Dynamic Web Service Selection," In Proceeding 13th International Conference, 2004.

[18] Y. Mou, J. Cao, S.S. Zhang, J.H. Zhang, "Interactive Web Service Choice-Making Based on Extended QoS Model," CIT, pp.1130-1134, 2005.

[19] D.A. Menasce, "QoS Issues in Web Services," IEEE Internet Computing, Vol.6, No.6, 2002.

[20] Stephan R.M., Hong Q. Y. and Marcel T., "Service Selection Based on Non-Functional Properties," NFPSLASOC 2007, LNCS, Springer, 2007.

[21] Satty T. L., 'The Analytic Hierarchy Process,' McGraw-Hill, 1980.

[22] Matthew et al. 'Reference Model for Service Oriented Architecture 1.0', OASIS Standard, 2006.

[23] Perez et. al, "Another Potential Shortcoming of AHP", Springer Berlin/Heidelberg, Vol.14, No.1, pp.91-111, 2006.

[24] Likert Rensis, "A Technique for the Measurement of Attitudes," Archives of Psychology, pp.1-55, 1932.

[25] 두화준, 이경호, "효율적인 서비스 조합을 위한 시맨틱 웹 서비 스 탐색", 한국정보과학회 논문집(B), Vol.32, No.1, pp.172-174, 2006.

[26] 김유경, 고병선, "QoS 기반 웹서비스 조합에서 신뢰성 평가를 통한 서비스 선택 기법", 정보과학회논문지 : 소프트웨어 및 응용, Vol.36, No.1, pp.1-9, 2009.

[27] 김건수, 이동훈, 박두경, 이지형, "사례 기반 추론을 이용한 서비 스 컴포지션 연구", 한국지능시스템학회 논문지, Vol.18, No.2, pp.175-182, 2008.

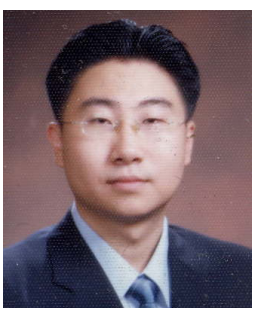

\section{김 지 혁}

e-mail : jhkim78@ssu.ac.kr

2003년 숭실대학교 컴퓨터학부(학사)

2005년 숭실대학교 컴퓨터학과(공학석사)

2005년 현 재 숭실대학교 컴퓨터학과 박사과정

관심분야:요구공학, 소프트웨어 유지보수, 소프트웨어 재사용, 서비스 지향 아키텍처, 오픈소스 소프트웨어, 소프트웨어 품질 보증 


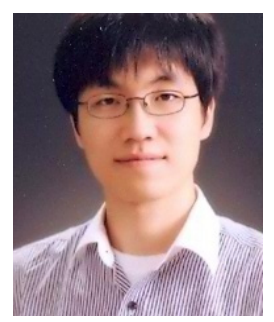

변 정 원

e-mail : Jimi01@ssu.ac.kr

2007년 숭실대학교 미디어학부(학사)

2008년 현 재 숭실대학교 컴퓨터학과 석사과정

관심분야 : 소프트웨어 요구공학, 소프트 웨어 유지보수, 소프트웨어 아키텍 처/프레임워크

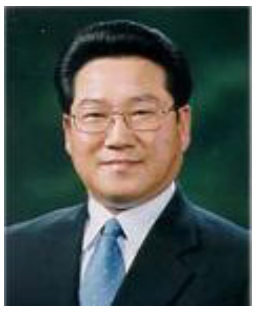

류 성 열

e-mail:syrhew@ssu.ac.kr

1981년 현 재 숭실대학교 컴퓨터학부 교수

1982년 1995년 숭실대학교 전자계산연구 소 및 중앙전자계산소 소장

1997년 1998년 George Mason University 객원 교수 1998년 2001년 숭실대학교 정보과학대학원 원장 2004년 현 재 한국품질재단 운영위원회 위원장 2006년 현 재 공정거래위원회 성과관리위원회위원 2008년 현 재 정보통신연구진흥원 비상임이사 관심분야: 요구공학, 소프트웨어 유지보수, 소프트웨어 재사용, 오픈소프 소프트웨어 